\section{Number OF COMPATIBLE PAIR OF ACTIONS fOR FinIte CYCLIC GROUPS OF P-POWER ORDER}

\author{
Mohammed Khalid Shahoodh, Mohd Sham Mohamad*, Yuhani \\ Yusof, Sahimel Azwal Sulaiman
}

Applied \& Industrial Mathematics (AIMs) Research Cluster, Faculty of Industrial Sciences \& Technology, Universiti Malaysia Pahang, Lebuhraya Tun Razak, 26300 Gambang, Kuantan, Pahang Darul Makmur, Malaysia
Article history

Received

17 July 2017

Received in revised form

27 April 2018

Accepted

15 May 2018

Published online

1 August 2018

*Corresponding author mohdsham@ump.edu.my

\section{Graphical abstract}

$(p-1) p^{\beta-1}+\sum_{k=1}^{\alpha-1}(p-1) p^{k-1}+\sum_{k=1}^{\alpha-1}(p-1) p^{k-1} \sum_{k=1}^{\alpha-1} \sum_{i=1}^{r}(p-1) p^{i-1}$

\begin{abstract}
The compatible actions played an important role before determining the nonabelian tensor product of groups. Different compatible pair of actions gives a different nonabelian tensor product even for the same group. The aim of this paper is to determine the exact number of the compatible pair of actions for the finite cyclic groups of p-power order where $p$ is an odd prime. By using the necessary and sufficient number theoretical conditions for a pair of the actions to be compatible with the actions that have p-power order, the exact number of the compatible pair of actions for the finite cyclic groups of $\mathrm{p}$-power order has been determined and given as a main result in this paper.
\end{abstract}

Keywords: Nonabelian tensor product, cyclic groups, automorphism group, compatible actions, number theory

\begin{abstract}
Abstrak
Tindakan yang serasi memainkan peranan penting sebelum menentukan kumpulan hasil darab tensor tak abelan. Pasangan tindakan serasi yang berbeza memberikan kumpulan hasil darab tensor yang berlainan walaupun daripada kumpulan yang sama. Objektif kajian ini adalah untuk menentukan jumlah sebenar pasangan tindakan serasi bagi kumpulan kitaran terhingga berperingkat kuasa $p$ dengan $p$ ialah nombor ganjil perdana. Dengan menggunakan syarat perlu dan cukup teori nombor untuk sepasang tindakan menjadi serasi dengan tindakan yang berperingkat kuasa $p$, jumlah sebenar pasangan tindakan serasi kumpulan kitaran terhingga berperingkat kuasa $p$ telah ditentukan dan diberikan sebagai hasil utama dalam makalah ini.

Kata kunci: Hasil darab tensor tak abelan, kumpulan kitaran, kumpulan automorfisma, tindakan serasi, teori nombor
\end{abstract}

(C) 2018 Penerbit UTM Press. All rights reserved 


\subsection{INTRODUCTION}

The nonabelian tensor product of groups has been discussed since 1984. Brown and Loday [1] were the first who introduced the concept of the nonabelian tensor product of groups with the compatible actions. This concept was originated in a connection with a generalized Van Kampen Theorem and its construction has its origins in the algebraic K-theory and also in the homotopy theory. Given two groups $G$ and $H$, then the nonabelian tensor product $G \otimes H$ is the group generated by $g \otimes h$ satisfying the following relations

$g g^{\prime} \otimes h=\left({ }^{g} g^{\prime} \otimes \otimes^{g} h\right)(g \otimes h)$ and $g \otimes h h^{\prime}=(g \otimes h)\left({ }^{h} g \otimes \otimes^{h} h^{\prime}\right)$

for all $g, g^{\prime} \in G$ and $h, h^{\prime} \in H$. If $G$ and $H$ be groups which act on each other and each of which acts on itself by conjugation and satisfying

$$
\left.{ }^{(g h)} g^{\prime}={ }^{g}\left({ }^{h}\left(g^{-1} g^{\prime}\right)\right) \text { and } l^{h} g\right) h^{\prime}={ }^{h}\left(g\left(^{h^{-1}} h^{\prime}\right)\right)
$$

for all $g, g^{\prime} \in G$ and $h, h^{\prime} \in H$, then the actions are said to be compatible.

A paper by Brown et al. [2] motivated many researchers to investigate several types of groups with the nonabelian tensor product and the nonabelian tensor square. Some known results with an overview on the nonabelian tensor product and literature was illustrated by Kappe [3]. Many researchers had studied the nonabelian tensor square $G \otimes G$, since the actions are trivial and easily to check the compatibility conditions. If $G \neq H$, then it's in needed to check the compatible of the actions when the actions are nontrivial.

Ellis and McDermott [4] investigated the nonabelian tensor product for different groups and checked the compatible conditions of nontrivial actions, but only focused on the quaternion groups of order up to 32. Visscher [5] continued the study on the nonabelian tensor product of $p$-power order, where $p$ is prime, and he focused on the cyclic groups. He computed some of the nonabelian tensor product of the cyclic groups of p-power order and gives a complete classification of the nonabelian tensor product of cyclic groups of 2-power order with mutual nontrivial actions of order two. Visscher [5] also provided necessary and sufficient conditions for a pair of finite cyclic groups of p-power order to act compatibility on each other.

Next, Mohamad [6] continued with Visscher's work and he gave new necessary and sufficient conditions for a pair of finite cyclic groups of $p$-power order to act compatibility on each other with the conditions are depend on the order of the action. Mohamad et al. [7] also provided the compatibility conditions and the nonabelian tensor product of the cyclic groups of order $p^{2}$ with the actions of order $p$.

Three years later, Sulaiman et al. [8] computed the compatible pair of nontrivial actions for some cyclic groups of 2-power order involving at least one of the actions having order greater than two. Sulaiman et al. [9] have studied the compatible pair of nontrivial actions for two same finite cyclic 2groups of 2-power order and provided the exact number of the compatible pair of nontrivial actions for such type groups with the actions that have a same order while Mohamad [10] give the number of compatible pair of actions for two same cyclic groups of 2-power order for all order of actions. Furthermore, Sulaiman et al. [11] give more general results which determined the maximum number of different nonabelian tensor product for the two finite cyclic groups of 2-power order by computing the exact number of the compatible pairs of actions for the given nonabelian tensor product for such type of groups.

Besides that, Shahoodh et al. [12] determined the compatible pair of nontrivial actions for cyclic groups of 3-power order. However, they gave some necessary and sufficient number of theoretical conditions for a pair of finite cyclic groups of 3-power order with nontrivial actions to act compatibly on each other with the actions that have order three and nine satisfy the compatibility conditions.

In this paper, the aim is to provide the exact number of the compatible pairs of actions for the finite cyclic groups of $p$-power order where $p$ is an odd prime. GAP software [13] is used to compute the compatible actions for the finite cyclic groups of $p$-power order where $p$ is an odd prime.

In section 2, some definitions and related results on the automorphism of cyclic groups of p-power order, number theory and compatible actions are given. Then, the number of the compatible pair of actions for the finite cyclic groups of $p$-power order where $p$ is an odd prime is given in Section 3. Lastly, the conclusions of this paper are given in Section 4.

\subsection{METHODOLOGY}

In this section, some definitions and related results on the automorphism of cyclic groups of p-power order, number theory and compatible actions are stated and will be used to prove the new results. Started with definitions of an action and compatible actions that are given as follows:

Definition 2.1 [5]

Let $G$ and $H$ be cyclic groups. An action of $G$ on $H$ is a mapping, $\Phi: G \rightarrow$ Aut $(H)$ such that

$$
\Phi\left(g g^{\prime}\right)(h)=\Phi(g)\left(\Phi\left(g^{\prime}\right)(h)\right)
$$

for all $g, g^{\prime} \in G$ and $h \in H$.

Definition 2.2 [5]

Let $G$ and $H$ be groups which act on each other and each of which acts on itself by conjugation. Then the actions are compatible if

$$
{ }^{(g h)} g^{\prime}={ }^{g}\left({ }^{h}\left(g^{-1} g^{\prime}\right)\right) \text { and }{ }^{\left({ }^{h} g\right)} h^{\prime}={ }^{h}\left(g^{g}\left(^{-1} h^{\prime}\right)\right)
$$

for all $g, g^{\prime} \in G$ and $h, h^{\prime} \in H$. 
Next, the automorphism of the finite cyclic group of p-power order is an isomorphic with a direct product of two finite cyclic groups as stated in following theorem.

Theorem 2.1 [14] Let $p$ be an odd prime and $\alpha \in \mathbb{Z}^{+}$. If $G$ is a cyclic group of order $p^{\alpha}$, then

$$
\begin{aligned}
\operatorname{Aut}\left(C_{p^{\alpha}}\right) & \cong C_{p-1} \times C_{p^{\alpha-1}} \cong C_{(p-1) p^{\alpha-1}} \\
\text { and }\left|\operatorname{Aut}\left(C_{p^{\alpha}}\right)\right|=\varphi\left(p^{\alpha}\right) & =(p-1) p^{\alpha-1} \text {. }
\end{aligned}
$$

Now, the Euler Phi-function for a given positive integer is stated in the following definition.

Definition 2.3 [15] Euler's $\phi$-function For $m \geq 1$, the Euler's Phi-function, denoted by $\varphi(m)$, is the number of the positive integers not exceeding $m$ that are relatively prime with $m$.

The following corollary gives that the trivial action is always compatible with any other action.

Corollary 2.1[5] Let $G$ and $H$ be groups. Furthermore, let $G$ act trivially on $H$. If $G$ is abelian, then for any action of $H$ on $G$ the mutual actions are compatible.

For the case of two finite cyclic groups of $p$-power order where $p$ is an odd prime, the theorem below stated the compatibility for the pair of the actions that have p-power order.

Theorem 2.2 [6] Let $G=\langle g\rangle \cong C_{p^{\alpha}}$ and $H=\langle h\rangle \cong C_{p^{\beta}}$ where $\quad \alpha, \beta \geq 2$. Furthermore, let $\sigma \in \operatorname{Aut}(G)$ with $|\sigma|=p^{k}$ where $k=1,2, \ldots, \alpha-1 \quad$ and $\sigma^{\prime} \in \operatorname{Aut}(H)$ with $\left|\sigma^{\prime}\right|=\mathrm{p}^{k^{\prime}}$ where $k^{\prime}=1,2, \ldots, \beta-1$. Then $\left(\sigma, \sigma^{\prime}\right)$ is a compatible pair if and only if $k+k^{\prime} \leq \min \{\alpha, \beta\}$.

In the next section, all the results for the number of the compatible pair of actions for the finite cyclic groups of $p$-power order where $p$ is an odd prime are given.

\subsection{RESULTS AND DISCUSSION}

In this section, the number of the compatible pair of actions is determined. According to the order of the actions between two finite cyclic groups of p-power order that satisfying the compatibility conditions, the number of the compatible pair of actions that have p-power order for such type groups has been determined. Since the finite cyclic groups of $p$-power order are considered, then an action of a group $G$ on a group $H$ is the homomorphism from $G$ to Aut $(H)$ and an action of a group $H$ on a group $G$ is the homomorphism from $H$ to $\operatorname{Aut}(G)$. Hence, the number of the automorphisms of such type groups needs to be find first. Thus, the number of the automorphisms for the finite cyclic groups of p-power order with respective order is given in the following proposition.

Proposition 3.1 Let $G=\langle x\rangle \cong C_{p^{\alpha}}$ be a finite cyclic group of $p$-power order with $p$ is an odd prime and $\alpha \in \mathbb{N}$. Then, there exist $(p-1) p^{k-1}$ automorphism of order $p^{k}$ where $k=1,2, \ldots, \alpha-1$.

Proof. Let $G=\langle x\rangle \cong C_{p^{\alpha}}$ be a finite cyclic group of $p$ power order with $\mathrm{p}$ is an odd prime and $\alpha \in \mathbb{N}$. Without loss of generality, suppose that $H$ be a finite cyclic $p$-subgroup of $G$ such that $|H|=p^{k}$ where $k=1,2, \ldots, \alpha-1$. Thus, each element that relatively prime with $p^{k}$ has an order $p^{k}$. Since $H$ be a cyclic subgroup, by Definition 2.3, we have $\varphi\left(p^{k}\right)=(p-1) p^{k-1}$ which give the number of the automorphisms that have order $P^{k}$.

Next, the number of the compatible pair of actions for the finite cyclic groups of $p$-power order is determined. Suppose that one of the actions has an order one, then the number of the compatible pair of actions for such type of groups given in the following proposition.

Proposition 3.2 Let $G \cong C_{p^{\alpha}}$ and $H \cong C_{p^{\beta}}$ be finite cyclic groups of $p$-power order. Furthermore, let $\rho \in \operatorname{Aut}(G)$ with $|\rho|=1$ and $\alpha, \beta \geq 1$. Then, the number of the compatible pair of actions is $(p-1) p^{\beta-1}$.

Proof. Let $G \cong C_{p^{\alpha}}$ and $H \cong C_{p^{\beta}}$ be finite cyclic groups of p-power order. Furthermore, let $\rho \in \operatorname{Aut}(G)$ with $|\rho|=1$ and $\alpha, \beta \geq 1$. By Corollary 2.1, when $G$ act trivially on $H$, then any action of $H$ on $G$ the mutual actions are compatible. Thus, by Theorem 2.1, $|\operatorname{Aut}(H)|=(p-1) p^{\beta-1}$ is the number of the compatible pairs of actions.

Now, the number of the compatible pair of actions for the two finite cyclic groups of $p$-power order where $p$ is an odd prime has been determined when one of the actions has an order $p^{k}$ where $k=1,2, \ldots, \alpha-1$. By using the necessary and sufficient number theoretical conditions for such type groups, the number of the compatible pair of actions for the specific value of $k$ is given as follows.

Proposition 3.3 Let $G \cong C_{p^{\alpha}}$ and $H \cong C_{p^{\beta}}$ be finite cyclic groups of p-power order. Furthermore, let $\rho \in \operatorname{Aut}(G)$ with $\quad|\rho|=p^{k} \quad$ where $k=1,2, \ldots, \alpha-1$ and $\rho^{\prime} \in \operatorname{Aut}(H)$ such that $\alpha, \beta \geq 3$. Then the number 
of the compatible pairs of actions is

$(p-1) p^{k-1}+(p-1) p^{k-1} \sum_{i=1}^{r}(p-1) p^{i-1}$ when $k=1,2, \ldots, \alpha-1$ and $r=\min \{\alpha, \beta\}-k$

Proof. Let $G \cong C_{p^{\alpha}}$ and $H \cong C_{p^{\beta}}$ be finite cyclic groups of p-power order. Furthermore, let $\rho \in \operatorname{Aut}(G)$ and $|\rho|=p^{k}$ where $k=1,2, \ldots, \alpha-1$ and $\rho^{\prime} \in \operatorname{Aut}(H)$ such that $\alpha, \beta \geq 3$. By Proposition 3.1, there are $(p-1) p^{k-1}$ automorphisms of order $p^{k}$ where $k=1,2, \ldots, \alpha-1$ and by Theorem 2.2, the actions are compatible with $|\rho|=1$ and $|\rho|=p^{k}$. Thus, we shall consider into two cases as follows:

Case I: Suppose that $|\rho|=1$, then by Corollary 2.1, when one of the actions is trivial, then the actions are compatible. Thus, there are $(p-1) p^{k-1}$ compatible pairs of actions under this case.

Case II: Suppose that $|\rho|=p^{k}$ where $k=1,2, \ldots, \alpha-1$ and by Theorem 2.2, the actions are compatible when $k+k^{\prime} \leq \min \{\alpha, \beta\}$. Hence, the number of the compatible pair of actions for every $k$ is the summation of the possibilities of the actions to be compatible, which are

$$
\begin{aligned}
& {\left[\left((p-1) p^{1-1}+(p-1) p^{2-1}+(p-1) p^{3-1}\right)\right.} \\
& \left.+\left((p-1) p^{1-1}+(p-1) p^{2-1}\right)+\left((p-1) p^{1-1}\right)\right]+\ldots \\
& +\left[(p-1) p^{\min \{\alpha, \beta\}-k-1}\right] \\
& =\sum_{i=1}^{r}(p-1) p^{i-1}
\end{aligned}
$$

where $r=\min \{\alpha, \beta\}-k$. By Proposition 3.1, there are $(p-1) p^{k-1}$ automorphisms of order $p^{k}$. Thus, there are $(p-1) p^{k-1} \sum_{i=1}^{r}(p-1) p^{i-1} \quad$ compatible pairs of actions under this case.

Therefore, in total there are $(p-1) p^{k-1}+(p-1) p^{k-1} \sum_{i=1}^{r}(p-1) p^{i-1}$ compatible pairs of actions with $r=\min \{\alpha, \beta\}-k$.

The following proposition gives the total number of the compatible pairs of actions for two finite cyclic groups of p-power order when one of the actions has the $p$-power order.

Proposition 3.4 Let $G=\langle x\rangle \cong C_{p^{\alpha}}$ and $H=\langle y\rangle \cong C_{p^{\beta}}$ where $\alpha, \beta \geq 3$. Furthermore, let $\rho$ and $\rho^{\prime}$ are elements of $\operatorname{Aut}(G)$ and $\operatorname{Aut}(H)$ respectively with $|\rho|=p^{k}$ where $k=1,2, \ldots, \alpha-1$. Then the total number of the compatible pairs of actions is $\sum_{k=1}^{\alpha-1}(p-1) p^{k-1}+\sum_{k=1}^{\alpha-1}(p-1) p^{k-1} \sum_{k=1}^{\alpha-1} \sum^{r}(p-1) p^{i-1} \quad$ when $k=1,2, \ldots, \alpha-1$ and $r=\min \{\alpha, \beta\}-k$.

Proof. Let $G=\langle x\rangle \cong C_{p^{\alpha}}$ and $H=\langle y\rangle \cong C_{p^{\beta}}$ where $\alpha, \beta \geq 3$. Furthermore, suppose that $\rho$ and $\rho^{\prime}$ are elements of $\operatorname{Aut}(G)$ and $\operatorname{Aut}(H)$ respectively with $|\rho|=p^{k}$ where $k=1,2, \ldots, \alpha-1$. By Proposition 3.3, there are

$$
(p-1) p^{k-1}+(p-1) p^{k-1} \sum_{i=1}^{r}(p-1) p^{i-1}
$$

compatible pairs of actions when $k=1,2, \ldots, \alpha-1$ and $r=\min \{\alpha, \beta\}-k$. Then in total if all $k$ 's are consider then the number of actions are given in the following

$$
\begin{aligned}
& \left((p-1) p^{1-1}+(p-1) p^{1-1} \sum_{i=1}^{r}(p-1) p^{i-1}\right) \\
+ & \left((p-1) p^{2-1}+(p-1) p^{2-1} \sum_{i=1}^{r}(p-1) p^{i-1}\right)+\cdots \\
+ & \left((p-1) p^{(\alpha-1)-1}+(p-1) p^{(\alpha-1)-1} \sum_{i=1}^{r}(p-1) p^{i-1}\right) \\
= & \sum_{k=1}^{\alpha-1}\left((p-1) p^{k-1}+(p-1) p^{k-1} \sum_{i=1}^{r}(p-1) p^{i-1}\right) \\
= & \sum_{k=1}^{\alpha-1}(p-1) p^{k-1}+\sum_{k=1}^{\alpha-1}(p-1) p^{k-1} \sum_{k=1}^{\alpha-1} \sum_{i=1}^{r}(p-1) p^{i-1} .
\end{aligned}
$$

Therefore, there are

$$
\sum_{k=1}^{\alpha-1}(p-1) p^{k-1}+\sum_{k=1}^{\alpha-1}(p-1) p^{k-1} \sum_{k=1}^{\alpha-1} \sum_{i=1}^{r}(p-1) p^{i-1}
$$

compatible pairs of actions.

In general, the number of the compatible pair of actions for the two finite cyclic groups of p-power order where $p$ is an odd prime for the given nonabelian tensor product $C_{p^{\alpha}} \otimes C_{p^{\beta}}$ is given in the following theorem.

Theorem 3.1 Let $G=\langle x\rangle \cong C_{p^{\alpha}}$ and $H=\langle y\rangle \cong C_{p^{\beta}}$ be finite cyclic groups of $p$-power order with $\alpha, \beta \geq 3$. Then, there exist

$$
(p-1) p^{\beta-1}+\sum_{k=1}^{\alpha-1}(p-1) p^{k-1}+\sum_{k=1}^{\alpha-1}(p-1) p^{k-1} \sum_{k=1}^{\alpha-1} \sum_{i=1}^{r}(p-1) p^{i-1}
$$

compatible pairs of actions when $k=1,2, \ldots, \alpha-1$ and $r=\min \{\alpha, \beta\}-k$.

Proof. Let $G=\langle x\rangle \cong C_{p^{\alpha}}$ and $H=\langle y\rangle \cong C_{p^{\beta}}$ be finite cyclic groups of $p$-power order. Furthermore, let $\rho \in \operatorname{Aut}(G)$ and $\rho^{\prime} \in \operatorname{Aut}(H)$ with $\alpha, \beta \geq 3$. The number of the compatible pairs of actions with specific order can be determined by separating into two cases 
which are $|\rho|=1$ and $|\rho|=p^{k}$ where $k=1,2, \ldots, \alpha-1$ as follows.

Case I: Suppose that $|\rho|=1$. By Proposition 3.2, when one of the actions is trivial, then the number of the compatible pair of actions is $(p-1) p^{\beta-1}$.

Case II: Suppose that $|\rho|=p^{k}$ where $k=1,2, \ldots, \alpha-1$. By Proposition 3.4, the number of the compatible pair of actions are $\sum_{k=1}^{\alpha-1}(p-1) p^{k-1}+\sum_{k=1}^{\alpha-1}(p-1) p^{k-1} \sum_{k=1}^{\alpha-1} \sum_{i=1}^{r}(p-1) p^{i-1}$ when $k=1,2, \ldots, \alpha-1$ and $r=\min \{\alpha, \beta\}-k$.

Hence, in total the number of the compatible pairs of actions for the finite cyclic groups of p-power order where $p$ is an odd prime are

$(p-1) p^{\beta-1}+\sum_{k=1}^{\alpha-1}(p-1) p^{k-1}+\sum_{k=1}^{\alpha-1}(p-1) p^{k-1} \sum_{k=1}^{\alpha-1} \sum_{i=1}^{r}(p-1) p^{i-1}$.

By using Theorem 3.1, the number of the compatible pair of actions for the finite cyclic groups of $p$-power order where $p$ is an odd prime has been determined. Next, an example is given to illustrate the number of the compatible pair of actions for a given two finite cyclic groups of $p$ power order.

Example 3.1 Let $\mathrm{G}=\mathrm{C}_{3^{3}}$ and $\mathrm{H}=\mathrm{C}_{3^{4}}$ be finite cyclic groups of 3-power order. Now, consider the actions of $G$ and $H$ act on each other such that ${ }^{g} h=h^{\prime}$ and ${ }^{n} g=g^{k}$ for $g \in G$ and $h \in H$ with $k, l \in \mathbb{Z}^{+}$. By Theorem 3.1 the number of the compatible pair of actions is $(3-1) 3^{4-1}+\sum_{k=1}^{2}(3-1) 3^{k-1}+\sum_{k=1}^{2}(3-1) 3^{k-1} \sum_{k=1}^{2} \sum_{i=1}^{3-k}(3-1) 3^{i-1}=90$.

Table 1 illustrated the number of the compatible pairs of actions for $C_{3^{3}} \otimes C_{3^{4}}$ given by using GAP software. From Table 1, there are 90 compatible pairs of actions for $\mathrm{C}_{3^{3}} \otimes \mathrm{C}_{3^{4}}$. Hence, the result from Theorem 3.1 is equivalent with the number of the compatible pairs of actions given in Table 1.

Next, the number of the compatible pair of actions for the finite cyclic groups of p-power order has been presented when the groups are the same. According to Theorem 3.1, the number of the compatible pairs of actions is given in Corollary 3.1.
Table 1 Compatible pair of actions for $C_{3^{3}} \otimes C_{3^{4}}$ with the actions ${ }^{g} h=h^{\prime}$ and ${ }^{h} g=g^{k}$ for $g \in\langle g\rangle \cong C_{3^{3}}$ and $h \in\langle h\rangle \cong C_{3^{4}}$ where $k, l \in \mathbb{Z}^{+}$.

\begin{tabular}{cccc}
\hline$|\rho|$ & $k$ & $\mid$ & $\left|\rho^{\prime}\right|$ \\
\hline 1 & 1 & 1 & 1 \\
1 & 1 & 2 & 54 \\
1 & 1 & 4 & 27 \\
1 & 1 & 5 & 54 \\
1 & 1 & 7 & 27 \\
1 & 1 & 8 & 18 \\
1 & 1 & 10 & 9 \\
1 & 1 & 11 & 54 \\
1 & 1 & 13 & 27 \\
1 & 1 & 14 & 54 \\
1 & 1 & 16 & 27 \\
1 & 1 & 17 & 18 \\
1 & 1 & 19 & 9 \\
1 & 1 & 20 & 54 \\
1 & 1 & 22 & 27 \\
1 & 1 & 23 & 54 \\
1 & 1 & 25 & 27 \\
1 & 1 & 26 & 6 \\
1 & 1 & 28 & 3 \\
1 & 1 & 29 & 54 \\
1 & 1 & 31 & 27 \\
1 & 1 & 32 & 54 \\
1 & 1 & 34 & 27 \\
1 & 1 & 35 & 18 \\
1 & 1 & 37 & 9 \\
1 & 1 & 38 & 54 \\
1 & 1 & 40 & 27 \\
1 & 1 & 41 & 54 \\
1 & 1 & 43 & 27 \\
1 & 1 & 44 & 18 \\
1 & 1 & 46 & 9 \\
1 & 1 & 47 & 54 \\
1 & 1 & 49 & 27 \\
1 & 1 & 50 & 54 \\
1 & 1 & 52 & 27 \\
1 & 1 & 53 & 6 \\
1 & 1 & 55 & 3 \\
1 & 1 & 56 & 54 \\
1 & 1 & 58 & 27 \\
1 & 1 & 59 & 54 \\
1 & 1 & 61 & 27 \\
1 & 1 & 62 & 18 \\
1 & 1 & 64 & 9 \\
1 & 1 & 65 & 54 \\
1 & 1 & 67 & 27 \\
\hline 1 & & &
\end{tabular}

\begin{tabular}{cccc}
\hline$|\rho|$ & $k$ & 1 & $\left|\rho^{\prime}\right|$ \\
\hline 1 & 1 & 68 & 54 \\
1 & 1 & 70 & 27 \\
1 & 1 & 71 & 18 \\
1 & 1 & 73 & 9 \\
1 & 1 & 74 & 54 \\
1 & 1 & 76 & 27 \\
1 & 1 & 77 & 54 \\
1 & 1 & 79 & 27 \\
1 & 1 & 80 & 2 \\
9 & 4 & 1 & 1 \\
9 & 4 & 28 & 3 \\
9 & 4 & 55 & 3 \\
9 & 7 & 1 & 1 \\
9 & 7 & 28 & 3 \\
9 & 7 & 55 & 3 \\
3 & 10 & 1 & 1 \\
3 & 10 & 10 & 9 \\
3 & 10 & 19 & 9 \\
3 & 10 & 28 & 3 \\
3 & 10 & 37 & 9 \\
3 & 10 & 46 & 9 \\
3 & 10 & 55 & 3 \\
3 & 10 & 64 & 9 \\
3 & 10 & 73 & 9 \\
9 & 13 & 1 & 1 \\
9 & 13 & 28 & 3 \\
9 & 13 & 55 & 3 \\
9 & 16 & 1 & 1 \\
9 & 16 & 28 & 3 \\
9 & 16 & 55 & 3 \\
3 & 19 & 1 & 1 \\
3 & 19 & 10 & 9 \\
3 & 19 & 19 & 9 \\
3 & 19 & 28 & 3 \\
3 & 19 & 37 & 9 \\
3 & 19 & 46 & 9 \\
3 & 19 & 55 & 3 \\
3 & 19 & 64 & 9 \\
3 & 19 & 73 & 9 \\
9 & 22 & 1 & 1 \\
9 & 22 & 28 & 3 \\
9 & 22 & 55 & 3 \\
9 & 25 & 1 & 1 \\
9 & 25 & 28 & 3 \\
9 & 25 & 55 & 3 \\
\hline & & &
\end{tabular}

Corollary 3.1 Let $G=H \cong C_{p^{\alpha}}$ be finite cyclic group of $\mathrm{p}$-power order with $\mathrm{p}$ is an odd prime and $\alpha \geq 3$. Then, there are

$$
(p-1) p^{\alpha-1}+\sum_{k=1}^{\alpha-1}(p-1) p^{k-1}+\sum_{k=1}^{\alpha-1}(p-1) p^{k-1} \sum_{k=1}^{\alpha-1} \sum_{i=1}^{\alpha-k}(p-1) p^{i-1}
$$

compatible pairs of actions. 
Proof. Let $G=H \cong C_{p^{\alpha}}$ be finite cyclic group of p-power order with $\mathrm{p}$ is an odd prime and $\alpha \geq 3$. Since $\mathrm{G}=H$, then $\alpha=\min \{\alpha, \alpha\}$. Therefore by Theorem 3.1, there are

$$
(p-1) p^{\alpha-1}+\sum_{k=1}^{\alpha-1}(p-1) p^{k-1}+\sum_{k=1}^{\alpha-1}(p-1) p^{k-1} \sum_{k=1}^{\alpha-1} \sum_{i=1}^{\alpha-k}(p-1) p^{i-1}
$$

compatible pairs of actions.

In particularly, the number of the compatible pair of nontrivial actions that have p-power order for nonabelian tensor product $C_{p^{\alpha}} \otimes C_{p^{\beta}}$ and $C_{p^{\beta}} \otimes C_{p^{\alpha}}$ are equal. This result is given in the following theorem.

Theorem 3.2 Let $G=\langle x\rangle \cong C_{p^{\alpha}}$ and $H=\langle y\rangle \cong C_{p^{\beta}}$ be finite cyclic groups of $p$-power order with $\alpha, \beta \geq 3$. Then the number of the compatible pair of nontrivial actions that have $p$-power order for the nonabelian tensor product $C_{p^{\alpha}} \otimes C_{p^{\beta}}$ and $C_{p^{\beta}} \otimes C_{p^{\alpha}}$ are equal.

Proof. Let $G=\langle x\rangle \cong C_{p^{\alpha}}$ and $H=\langle y\rangle \cong C_{p^{\beta}}$ be finite cyclic groups of $\mathrm{p}$-power order with $\alpha, \beta \geq 3$. By Proposition 3.4, there are $\sum_{k=1}^{\alpha-1}(p-1) p^{k-1} \sum_{k=1}^{\alpha-1} \sum_{i=1}^{r}(p-1) p^{i-1}$ compatible pairs of nontrivial actions when $k=1,2, \ldots, \alpha-1 \quad$ and $\quad r=\min \{\alpha, \beta\}-k$ since $r=\min \{\alpha, \beta\}-k=\min \{\beta, \alpha\}-k$, then for any given nonabelian tensor product $C_{p^{\alpha}} \otimes C_{p^{\beta}}$ or $C_{p^{\beta}} \otimes C_{p^{\alpha}}$, the number of compatible pairs of nontrivial actions are same.

\subsection{CONCLUSION}

The exact number of the compatible pair of actions between a pair of the finite cyclic 2-groups of $p$ power order where $p$ is an odd prime has been given. By using the necessary and sufficient conditions for the pair of the actions to be compatible with the actions that have p-power order satisfying the compatibility conditions, the number of the compatible pair of actions is determined. This paper also gives the number of the compatible pair of actions for the same such type groups with the actions that have p-power order. The obtained results gives that the number of the compatible pair of nontrivial actions for the given nonabelian tensor product for such type groups are equal.

\section{Acknowledgement}

This research is fully supported by UMP Research Grant, RDU1703265. The authors fully acknowledged Universiti Malaysia Pahang for the approved fund which makes this important research viable and effective.

\section{References}

[1] Brown, R. and Loday, J. L. 1984. Excision homotopique en basse dimension, CR Acad. Sci. Paris SI Math. 298(15): 353356.

[2] Brown, R., Johnson, D. L. and Robertson, E. F. 1987. Some Computations of Non-Abelian Tensor Products of Groups. Journal of Algebra. 11 (1):177-202.

[3] Kappe, L. C. 1997. Nonabelian Tensor Products of Groups: The Commutator Connection. London Mathematical Society Lecture Note Series. 447-454.

[4] Ellis, G. and McDermott, A. 1998. Tensor Products of Prime Power Groups. Journal of Pure and Applied Algebra. 132(2): 119-128.

[5] Visscher, M. P. 1998. On the Nonabelian Tensor Product of Groups. Dissertation. State University of New York.

[6] Mohamad, M. S. 2012. Compatibility Conditions and Nonabelian Tensor Products of Finite Cyclic Groups of $p$ Power Order. PhD Thesis. Universiti Teknologi Malaysia.

[7] Mohamad, M. S., Sarmin, N. H., Ali, N. M. M., and Kappe, L. C. 2012. The Computation of the Nonabelian Tensor Product of Cyclic Group of Order $p^{2}$. Journal Teknologi. 57(1).

[8] Sulaiman, S. A., Mohamad, M. S., Yusof, Y., Sarmin, N. H., Ali, M. M. N., Ken, T. L., and Ahmad, T. 2015. Compatible Pair of Nontrivial Actions for Some Cyclic Groups of 2Power Order. The $2^{\text {nd }}$ ISM International Statistical Conference 2014 (ISM-II): Empowering the Applications of Statistical and Mathematical Sciences. AlP Publishing. 1643: 700-705.

[9] Sulaiman, S. A., Mohamad, M. S., Yusof, Y and Shahoodh, M. K. 2016. Compatible Pair of Nontrivial Action for Finite Cyclic 2-Groups. The National Conference for Postgraduate Research 2016, Universiti Malaysia Pahang. p005: 39-42.

[10] Mohamad, M. S., Sulaiman, S. A., Yusof, Y. and Shahoodh, M. K. 2017. Compatible Pair of Actions for Two Same Cyclic Groups of 2-Power Order. IOP Conf. Series: Journal of Physics: Conf. Series 890 (2017) 012120

[11] Sulaiman, S. A., Mohamad, M. S., Yusof, Y. and Shahoodh, K. M. 2017. The Number of Compatible Pair of Actions for Cyclic Groups of 2-Power Order. International Journal of Simulation, System, Sciences and Technology. 18(4): 14738031.

[12] Shahoodh, K. M., Mohamad, M. S., Yusof, Y. and Sulaiman, S. A. 2017. Number of Compatible Pair of Actions for Finite Cyclic Groups of 3-Power Order. International Journal of Simulation, System, Sciences and Technology. 18(4): 14738031.

[13] http://www.gap-system.org.The GAP Group, GAP-Groups, Algorithm and programming. Version 4.7, 2015.

[14] Dummit, D. S and Foote, R. M. 2004. Abstract Algebra. USA: John Wiley and Sons.

[15] Burton, D. 2005. Elementary Number Theory. Sixth Edition. USA: McGraw Hill. 